\title{
A Neurobehavioral Model of Flexible Spatial Language Behaviors Supplemental Material
}

\author{
John Lipinski, Sebastian Schneegans, Yulia Sandamirskaya, \\ John P. Spencer, and Gregor Schöner
}

\section{Formal description of the computational model}

We use a unique three-letter index for each field or group of notes, which is appended to all associated variables and model parameters: csf (color-space fields), $c o l$ (color term nodes), tar (target field), ref (reference field), $t r n$ (transformation field), $o b j$ (object-centered field), $s p r$ (spatial relation nodes) and $s p t$ (spatial term nodes). Parameters of projections carry two indices, the first designating its origin, the second its target.

\section{Extraction of color information}

The visual input to the system is a camera image $I(152 \times 120$ pixels $)$, from which a binarized map $I^{\mathrm{c}}$ is extracted for each color $c \in C, C=\{$ red, green, blue $\}$. Color extraction uses the HSV color space and is performed according to the equation:

$$
I^{\mathrm{c}}(x, y)= \begin{cases}1 & \text { if } h(I(x, y)) \in H^{\mathrm{c}} \wedge s(I(x, y))>\theta_{\mathrm{s}} \wedge v(I(x, y))>\theta_{\mathrm{v}} \\ 0 & \text { otherwise }\end{cases}
$$

The ranges of hue values for the different colors are $H^{\text {red }}=\left[0^{\circ}, 60^{\circ}\right) \cup\left[300^{\circ}, 360^{\circ}\right), H^{\text {green }}=\left[60^{\circ}, 180^{\circ}\right)$, $H^{\text {blue }}=\left[180^{\circ}, 300^{\circ}\right)$, and the thresholds for saturation and value are $\theta_{\mathrm{s}}=0.25$ and $\theta_{\mathrm{v}}=0.25$, respectively (with ranges of values $h \in\left[0^{\circ}, 360^{\circ}\right), s \in[0,1]$ and $v \in[0,1]$ ).

\section{Mathematics of field interactions}

Each dynamic neural field as well as each set of dynamic nodes is described as an activity distribution $U$. The field output $O$ at each position is determined through a logistic function $f$ with steepness parameter $\beta$, yielding e. g. for a two-dimensional field

$$
O(x, y)=f(U(x, y))=\frac{1}{1+\exp (-\beta U(x, y))} .
$$

Lateral interactions within a field are mediated by interaction kernels, which are either of the difference-ofGaussians type, indicated by a kernel function $D$, or have a Gaussian excitatory part and a homogeneous (global) inhibition, with a kernel function $H$ :

$$
\begin{aligned}
& D(x, y)=\frac{e}{2 \pi \sigma^{\mathrm{exc} 2}} \exp \left(-\frac{x^{2}+y^{2}}{2 \sigma^{\mathrm{exc} 2}}\right)-\frac{i}{2 \pi \sigma^{\text {inh }^{2}}} \exp \left(-\frac{x^{2}+y^{2}}{2 \sigma^{\mathrm{inh}^{2}}}\right) \\
& H(x, y)=\frac{e}{2 \pi \sigma^{2}} \exp \left(-\frac{x^{2}+y^{2}}{2 \sigma^{2}}\right)-i
\end{aligned}
$$

The projections between fields either use a kernel $H$ with global inhibition, or are smoothed by a simple Gaussian interaction kernel $G$ of the form

$$
G(x, y)=\frac{e}{2 \pi \sigma^{2}} \exp \left(-\frac{x^{2}+y^{2}}{2 \sigma^{2}}\right) .
$$


The weights $e, i$ and widths $\sigma^{\text {exc }}, \sigma^{\text {inh }}$ or $\sigma$ are parameters specified seperately for each kernel. The interaction kernels are convolved with the field output, with the two-dimensional convolution generically defined as

$$
\left.[O * G](x, y)=\iint O\left(x-x^{\prime}, y-y^{\prime}\right)\right) G\left(x^{\prime}, y^{\prime}\right) d x^{\prime} d y^{\prime}
$$

\section{Field equations}

For each color $c \in C$ a color-space field $U_{\text {csf }}^{\mathrm{c}}(x, y)$ is defined over the space of image positions, governed by the differential equation

$$
\begin{aligned}
\tau \dot{U}_{\mathrm{csf}}^{\mathrm{c}}(x, y)= & -U_{\mathrm{csf}}^{\mathrm{c}}(x, y)+h_{\mathrm{csf}}+e_{\mathrm{I}, \mathrm{csf}} I^{\mathrm{c}}(x, y)+e_{\mathrm{col}, \mathrm{csf}} O_{\mathrm{col}}(c) \\
& +\left[O_{\mathrm{tar}} * H_{\mathrm{tar}, \mathrm{csf}}\right](x, y)+\left[O_{\mathrm{ref}} * H_{\mathrm{ref}, \mathrm{csf}}\right](x, y)+\left[O_{\mathrm{csf}}^{\mathrm{c}} * D_{\mathrm{csf}}\right](x, y)
\end{aligned}
$$

with time constant $\tau$ and resting level $h_{\text {csf }}$. The activity of the associated color term node, $U_{\text {col }}(c)$, follows the equation

$$
\begin{aligned}
\tau \dot{U}_{\mathrm{col}}(c)= & -U_{\mathrm{col}}(c)+h_{\mathrm{col}}+e_{\mathrm{csf}, \mathrm{col}} \iint O_{\mathrm{csf}}^{\mathrm{c}}(x, y) d x d y \\
& +e_{\mathrm{col}} O_{\mathrm{col}}(c)-i_{\mathrm{col}} \sum_{c^{\prime} \in C} O_{\mathrm{col}}\left(c^{\prime}\right)+m_{\mathrm{col}}(c)+b_{\mathrm{col}} .
\end{aligned}
$$

Each node can receive specific task input $m_{\mathrm{col}}(c)$ (either zero or $m_{\mathrm{col}}^{\text {set }}=5$ if the color is selected by the task) and a global boost $b_{\text {col }}$ (either zero or $b_{\text {col }}^{\text {select }}=4$ if a color response is queried). A color term is given as response if the node output exceeds $\theta_{\text {col }}=0.75$.

The field equation for the target field $U_{\text {tar }}$ is

$$
\begin{aligned}
\tau \dot{U}_{\mathrm{tar}}(x, y)= & -U_{\mathrm{tar}}(x, y)+h_{\mathrm{tar}}+\sum_{c \in C}\left[O_{\mathrm{csf}}^{\mathrm{c}} * G_{\mathrm{csf}, \mathrm{tar}}\right](x, y)+\left[O_{\mathrm{trn}}^{\mathrm{tar}} * G_{\mathrm{trn}, \mathrm{tar}}\right](x, y) \\
& -\left[O_{\mathrm{ref}} * G_{\mathrm{ref}, \operatorname{tar}}\right](x, y)+\left[O_{\mathrm{tar}} * H_{\mathrm{tar}}\right](x, y)+b_{\mathrm{tar}},
\end{aligned}
$$

with the transformation field output summed over all reference positions,

$$
O_{\operatorname{trn}}^{\operatorname{tar}}(x, y)=\iint O_{\operatorname{trn}}(x, y, u, v) d u d v .
$$

The global boost input $b_{\mathrm{tar}}$ takes the values $b_{\mathrm{tar}}^{\text {select }}=4$ to select the target location, $b_{\mathrm{tar}}^{\text {hold }}=2$ to hold a location, and zero otherwise. The reference field is defined completely analogous to the target field (with transformation field output summed over all target positions):

$$
\begin{aligned}
\tau \dot{U}_{\mathrm{ref}}(x, y)= & -U_{\mathrm{ref}}(x, y)+h_{\mathrm{ref}}+\sum_{c \in C}\left[O_{\mathrm{csf}}^{\mathrm{c}} * G_{\mathrm{csf}, \mathrm{tar}}\right](x, y)+\left[O_{\mathrm{trn}}^{\mathrm{ref}} * G_{\mathrm{trn}, \mathrm{tar}}\right](x, y) \\
& -\left[O_{\mathrm{tar}} * G_{\mathrm{ref}, \mathrm{tar}}\right](x, y)+\left[O_{\mathrm{ref}} * H_{\mathrm{ref}}\right](x, y)+b_{\mathrm{ref}} \\
O_{\mathrm{trn}}^{\mathrm{ref}}(u, v)= & \iint O_{\mathrm{trn}}(x, y, u, v) d x d y
\end{aligned}
$$

The transformation field is defined over the space of target positions (here $x, y$ ) times reference positions $(u, v)$, with field equation

$$
\begin{aligned}
\tau \dot{U}_{\mathrm{trn}}(x, y, u, v)= & -U_{\mathrm{trn}}(x, y, u, v)+h_{\mathrm{trn}}+\left[O_{\mathrm{trn}} * G_{\mathrm{tar}, \mathrm{trn}}\right](x, y) \\
& +\left[O_{\mathrm{ref}} * G_{\mathrm{tar}, \mathrm{trn}}\right](u, v)+\left[O_{\mathrm{obj}} * G_{\mathrm{obj}, \mathrm{trn}}\right](x-u, y-v) \\
& -i_{\mathrm{trn}} \iiint \int O_{\mathrm{trn}}\left(x^{\prime}, y^{\prime}, u^{\prime}, v^{\prime}\right) d x^{\prime} d y^{\prime} d u^{\prime} d v^{\prime} .
\end{aligned}
$$


The object-centered field is defined over the space of relative target positions, covering twice the size of the target and reference fields, with

$$
\begin{aligned}
\tau \dot{U}_{\mathrm{obj}}(x, y)= & -U_{\mathrm{obj}}(x, y)+h_{\mathrm{obj}}+\left[O_{\mathrm{trn}}^{\mathrm{obj}} * G_{\mathrm{trn}, \mathrm{obj}}\right](x, y) \\
& +e_{\mathrm{spr}, \mathrm{obj}} \sum_{s \in S} O_{\mathrm{spr}}(s) W^{\mathrm{s}}(x, y)-i_{\mathrm{obj}} \iint O_{\mathrm{obj}}\left(x^{\prime}, y^{\prime}\right) d x^{\prime} d y^{\prime}+b_{\mathrm{obj}}
\end{aligned}
$$

The global boost $b_{\mathrm{obj}}$ takes the values zero or $h_{\mathrm{obj}}^{\text {high }}=1$. A semantic weight pattern $W^{\mathrm{s}}$ is defined for each spatial term $s \in S, S=\{$ above, below, left, right $\}$ :

$$
W^{\mathrm{s}}(x, y)=w^{\mathrm{s}}\left(w_{\mathrm{G}} G^{\mathrm{s}}(x, y)+w_{\mathrm{L}} L^{\mathrm{s}}(x, y)\right)
$$

(with weights $w^{\text {above }}=w^{\text {below }}=1$ and $w^{\text {left }}=w^{\text {right }}=0.875$ for normalization, $w_{\mathrm{G}}=0.525$ and $w_{\mathrm{L}}=$ $0.475)$. The first component $G^{\mathrm{s}}$ is a Gaussian in polar coordinates,

$$
G^{\mathrm{s}}(x, y)=\exp \left(-\frac{\left|\phi(x, y)-\phi^{\mathrm{s}}\right|^{2}}{2 \psi^{2}}-\frac{(r(x, y)-\rho)^{2}}{2 \lambda^{2}}\right),
$$

with $\phi(x, y)$ and $r(x, y)$ being the polar coordinates (angle and distance) corresponding to the Cartesian coordinates $(x, y)$, and the norm $|$.$| denoting the angular difference shifted to the range [-\pi, \pi)$. The angular centers $\phi^{\mathrm{s}}$ are set for each term such that the Gaussian is directed at the appropriate direction from the origin (located at the center of the field), namely $\phi^{\text {above }}=\frac{\pi}{2}$, $\phi^{\text {below }}=-\frac{\pi}{2}, \phi^{\text {left }}=-\pi, \phi^{\text {right }}=0$. The further parameters are $\psi=0.25, \rho=15$ and $\lambda=100$. The second component is a sigmoid of the position along one axis. For the relation above it is given by

$$
L^{\text {above }}(x, y)=\frac{1}{1+\exp \left(-\beta_{L} y\right)},
$$

for the relations below, left and right, the $y$ on the right-hand side has to be changed to $-y,-x$ and $x$, respectively. The steepness is $\beta_{L}=0.5$.

There is one spatial relation node $U_{\text {spr }}(s)$ for each $s \in S$, with field equation

$$
\begin{aligned}
\tau \dot{U}_{\mathrm{spr}}(s)= & -U_{\mathrm{spr}}(s)+h_{\mathrm{spr}}+e_{\mathrm{obj}, \mathrm{spr}} \iint O_{\mathrm{obj}}(x, y) W^{\mathrm{s}}(x, y) d x d y \\
& +e_{\mathrm{spt}, \mathrm{spr}} O_{\mathrm{spt}}(s)-i_{\mathrm{spt}, \mathrm{spr}} \sum_{s^{\prime} \in S} O_{\mathrm{spt}}\left(s^{\prime}\right)+e_{\mathrm{spr}} O_{\mathrm{spr}}(s)-i_{\mathrm{spr}} \sum_{s^{\prime} \in S} O_{\mathrm{spr}}\left(s^{\prime}\right),
\end{aligned}
$$

and a corresponding spatial term node $U_{\text {spt }}(s)$,

$$
\begin{aligned}
\tau \dot{U}_{\mathrm{spt}}(s)= & -U_{\mathrm{spt}}(s)+h_{\mathrm{spt}}+e_{\mathrm{spr}, \mathrm{spt}} O_{\mathrm{spr}}(s) \\
& +e_{\mathrm{spt}} O_{\mathrm{spt}}(s)-i_{\mathrm{spt}} \sum_{s^{\prime} \in S} O_{\mathrm{spt}}\left(s^{\prime}\right)+b_{\mathrm{spt}}+m_{\mathrm{spt}}(s),
\end{aligned}
$$

with specific task input $m_{\mathrm{spt}}(s)$ (either zero or $m_{\mathrm{spt}}^{\mathrm{set}}=5$ ), global boost input $b_{\mathrm{spt}}\left(\right.$ either zero or $b_{\mathrm{spt}}^{\text {select }}=4$ ), and a response threshold $\theta_{\text {spt }}=0.75$.

\section{Discrete implementation and activation noise}

For the simulations, field activities are evaluated only at fixed, regularly spaced positions, and the change over time is determined using the Euler algorithm. The fields $U_{\mathrm{csf}}^{\mathrm{c}}, U_{\mathrm{tar}}$ and $U_{\text {ref }}$ are sampled with $152 \times 120$ discrete units each, $U_{\text {trn }}$ with a reduced resolution of $19 \times 15$ units for both target and reference dimensions, $U_{\text {obj }}$ with $303 \times 239$ units (using the same resolution as $U_{\mathrm{tar}}$, but covering twice the space). All width parameters of interaction kernels are given in field units (for projections between fields, in units of the target if the fields have different resolutions).

For Demonstration 5, uncorrelated, normally distributed noise is added to the activities of all fields and nodes in each time step, scaled with a noise strength $q$ :

$$
\tilde{U}(x, y)=U(x, y)+q \mathcal{N}(0,1)
$$

Computation then continues with these noisy field activations. Noise strength is $q=0.1$ for all fields except for the two sets of output nodes, where it is reduced to $q_{\mathrm{col}}=q_{\mathrm{spt}}=0.025$. 
Table A.1

Field parameters and lateral interaction strength

\begin{tabular}{|c|c|c|c|c|c|c|c|c|c|}
\hline \multicolumn{3}{|c|}{ Resting level } & \multicolumn{2}{|c|}{ Sigmoid steepness } & \multicolumn{2}{|c|}{ Lateral excitation } & \multicolumn{3}{|c|}{ Lateral inhibition } \\
\hline$h_{\mathrm{csf}}$ & $=$ & -2 & $\beta_{\mathrm{csf}}$ & $=4$ & $e_{\mathrm{csf}}$ & $=2.5$ & $i_{\mathrm{csf}}$ & $=$ & 10 \\
\hline$h_{\mathrm{col}}$ & $=$ & -4 & $\beta_{\mathrm{col}}$ & $=4$ & $e_{\mathrm{col}}$ & $=2.5$ & $i_{\mathrm{col}}$ & $=$ & 2 \\
\hline$h_{\mathrm{tar}}$ & $=$ & -4 & $\beta_{\operatorname{tar}}$ & $=4$ & $e_{\operatorname{tar}}$ & $=10$ & $i_{\text {tar }}$ & $=$ & 0.02 \\
\hline$h_{\text {ref }}$ & $=$ & -4 & $\beta_{\text {ref }}$ & $=4$ & $e_{\text {ref }}$ & $=10$ & $i_{\text {ref }}$ & $=$ & 0.02 \\
\hline$h_{\mathrm{trn}}$ & $=$ & -2 & $\beta_{\mathrm{trn}}$ & $=4$ & & & $i_{\text {trn }}$ & $=$ & 0.0075 \\
\hline$h_{\text {obj }}$ & $=$ & -1 & $\beta_{\text {obj }}$ & $=2.5$ & & & $i_{\text {obj }}$ & $=$ & 0.000175 \\
\hline$h_{\mathrm{spr}}$ & $=$ & -1.95 & $\beta_{\mathrm{spr}}$ & $=2$ & $e_{\mathrm{spr}}$ & $=0.25$ & $i_{\mathrm{spr}}$ & $=$ & 1.25 \\
\hline$h_{\mathrm{spt}}$ & $=$ & -4 & $\beta_{\mathrm{spt}}$ & $=4$ & $e_{\mathrm{spr}}$ & $=2.5$ & $i_{\mathrm{spr}}$ & $=$ & 4 \\
\hline
\end{tabular}

The time constant for all fields is $\tau=5$, the lateral excitation width (where applicable) is $\sigma_{\mathrm{csf}}^{\text {exc }}=\sigma_{\mathrm{tar}}=$ $\sigma_{\text {ref }}=4$, the lateral inhibition width of the color-space fields is $\sigma_{\mathrm{csf}}^{\mathrm{inh}}=10$.

Table A.2

Parameters of interactions between fields

\begin{tabular}{|c|c|c|c|c|c|c|c|}
\hline \multicolumn{3}{|c|}{ Excitatory weight } & \multicolumn{2}{|c|}{ Kernel width } & \multicolumn{3}{|c|}{ Inhibitory weight } \\
\hline$e_{\mathrm{I}, \mathrm{csf}}$ & $=$ & 2 & & & & & \\
\hline$e_{\mathrm{csf}, \mathrm{col}}$ & $=$ & 0.01 & & & & & \\
\hline$e_{\mathrm{csf}, \mathrm{tar}}$ & $=$ & 6 & $\sigma_{\mathrm{csf}, \mathrm{tar}}$ & $=4$ & & & \\
\hline$e_{\mathrm{csf}, \text { ref }}$ & $=$ & 6 & $\sigma_{\mathrm{csf}, \text { ref }}$ & $=4$ & & & \\
\hline$e_{\mathrm{col}, \mathrm{csf}}$ & $=$ & 1 & & & & & \\
\hline$e_{\mathrm{tar}, \mathrm{csf}}$ & $=$ & 4 & $\sigma_{\mathrm{tar}, \mathrm{csf}}$ & $=15$ & $i_{\mathrm{tar}, \mathrm{csf}}$ & $=$ & 0.0005 \\
\hline$e_{\mathrm{tar}, \mathrm{trn}}$ & $=$ & 5 & $\sigma_{\mathrm{tar}, \operatorname{trn}}$ & $=1$ & & & \\
\hline$i_{\mathrm{tar}, \text { ref }}$ & $=$ & 1.5 & $\sigma_{\text {tar, ref }}$ & $=4$ & & & \\
\hline$e_{\text {ref, } \mathrm{csf}}$ & $=$ & 4 & $\sigma_{\text {ref, csf }}$ & $=15$ & $i_{\mathrm{ref}, \mathrm{csf}}$ & $=$ & 0.0005 \\
\hline \multirow[t]{2}{*}{$e_{\mathrm{ref}, \mathrm{trn}}$} & $=$ & 5 & $\sigma_{\text {ref, trn }}$ & $=1$ & & & \\
\hline & & & $\sigma_{\text {ref, } \operatorname{tar}}$ & $=4$ & $i_{\mathrm{ref}, \operatorname{tar}}$ & $=$ & 1.5 \\
\hline$e_{\mathrm{trn}, \operatorname{tar}}$ & $=$ & 0.175 & $\sigma_{\text {trn, tar }}$ & $=4$ & & & \\
\hline$e_{\mathrm{trn}, \text { ref }}$ & $=$ & 0.175 & $\sigma_{\text {trn, } \operatorname{tar}}$ & $=4$ & & & \\
\hline$e_{\mathrm{trn}, \mathrm{obj}}$ & $=$ & 0.75 & $\sigma_{\text {trn, obj }}$ & $=4$ & & & \\
\hline$e_{\mathrm{obj}, \mathrm{trn}}$ & $=$ & 1.5 & $\sigma_{\mathrm{obj}, \mathrm{trn}}$ & $=4$ & & & \\
\hline$e_{\mathrm{obj}, \mathrm{spr}}$ & $=$ & 0.00215 & & & & & \\
\hline$e_{\mathrm{spr}, \mathrm{obj}}$ & $=$ & 1.0 & & & & & \\
\hline$e_{\mathrm{spr}, \mathrm{spt}}$ & $=$ & 2 & & & & & \\
\hline$e_{\mathrm{spt}, \mathrm{spr}}$ & $=$ & 4 & & & $i_{\text {spt, spr }}$ & $=$ & 2.5 \\
\hline
\end{tabular}

